\title{
Infant Feeding Practices in The Klang Valley, Malaysia
}

\author{
N. S. Bachtiar ${ }^{1}$, R. Hussain ${ }^{2}$, S. A. Lanham-New ${ }^{1}$ and K. Horton ${ }^{1}$ \\ ${ }^{1}$ Faculty of Health and Medical Sciences, University of Surrey, GU2 $7 X H$ and ${ }^{2}$ Faculty of Medicine, \\ University of Malaya, Malaysia
}

Breastfeeding has always been the most optimum nutrition to babies. Despite its numerous benefits to the baby, mother and the society, the breastfeeding rate and duration is still low in most developing countries including Malaysia. The latest survey by the Malaysian's Ministry of Health in 2006 showed that the breastfeeding rate at 4 months was only $32 \%$ and even lower at 6 months $(19.4 \%)^{(1)}$. The aim of this study is to show the current breastfeeding rate in the Klang Valley, Malaysia and looking at the factors involved in the early breastfeeding cessation. Mothers who delivered their babies were invited to answer a 55-point questionnaire based from the United State's Infant Feeding Practices Study II (IFPS II) ${ }^{(2)}$ via face to face or telephone call. Some of the questions include the type of infant feeding method, influence from husband, family and the media regarding the decision of infant feeding method, problems in breastfeeding, reasons of breastfeeding cessation and social and health supports received by the mother. The study showed that $93 \%$ of the mothers $(n=259)$ initiated breastfeeding in the hospital. However only $33 \%$ of them were exclusively breastfeeding their babies at 4 months and $21 \%$ of the mothers were exclusively breastfeeding their babies at 6 months old. Forty two percent of the mothers said that husbands played an important role in the decision making of the infant feeding type, while $34 \%$ agreed that grandmothers had a strong influence in the same decision making. Fifty four percent of the mothers said that having a low milk supply as the main reason to stop breastfeeding, while $35 \%$ of them had to stop breastfeeding because they were returning to work. The remaining $11 \%$ had other reasons such as baby refused to breastfeed, discouraged by family members and mother or baby had a health problem. Majority of the mothers had some health support to breastfeed from the medical staff and about $45 \%$ did not received any support from the family members. Even though the Malaysian Government has been actively promoting breastfeeding to all its hospital, the breastfeeding pick up rate is still unsatisfactory. The role of family members and the society in supporting mothers to breastfeed has been undermined and could be one of the main reasons for the low and short breastfeeding rate and duration. Less mothers in the Klang Valley Malaysia were successful in breastfeeding their babies due to a variety of reasons including failure to get enough support from family members and society. This showed that mother's knowledge alone is insufficient to ensure breastfeeding success. Effort must be made to help mothers to successfully breastfeed their babies such as educating the immediate family members (husband, grandparents, siblings), providing sufficient facilities for breastfeeding at the work place and the formation of more breastfeeding support group.

1. Salim F, Hassan Nudin SS, Muhammad Ismail HI \& Aris T (2007) Infant Feeding: The Third National Health and Morbidity Survey 2006 (NHMS III). Kuala Lumpur: Ministry of Health Malaysia.

2. Sara BF, Laurence MG-S \& Tonse NKR (2008) Infant Feeding and Care Practices in the United States: Results From the Infant Feeding Practices Study II. Pediatrics 122, S25-S27. 